\title{
A COMPACT LOW-POWER MEM RESONATOR-BASED ANALOG TO DIGITAL CONVERTER WITH FEEDTHROUGH SIGNAL CANCELLATION
}

\author{
Sally Ahmed*, Xuecui Zou, and Hossein Fariborzi.
}

Computer, Electrical, and Mathematical Science and Engineering Division (CEMSE), King Abdullah University of Science and Technology (KAUST), Saudi Arabia.

\begin{abstract}
In this paper, the design and experimental results of a compact microelectromechanical (MEM) resonator-based analog to digital converter (ADC) are presented. The proposed N-bit ADC design produces the output bits using only $\mathrm{N}$ resonators. In addition, the device is designed in a symmetric way to cancel the feedthrough signal using a differential amplifier. While the measured ADC's sampling rate is $58 \mathrm{~S} / \mathrm{s}$, consuming $3.897 \mathrm{pJ} /$ conversion step, we show that by down-scaling the resonator's dimensions, $\mathrm{MHz}$ sampling rates and femto-joules energy consumption per conversion step are attainable, making resonator-based ADCs an attractive contender for mediumspeed ultra-low power applications.
\end{abstract}

\section{KEYWORDS}

Data converters, Analog to digital converter, MEMS resonators, clamped-clamped beam, electrostatic softening effect, parasitic feedthrough signal.

\section{INTRODUCTION}

The internet of things (IoT) era has allowed the communication and sharing of data between billions of smart objects equipped with different sensors, electronic chips, and communication modules. Data converters such as analog to digital (ADC) and digital to analog (DAC) converters are among the most essential sensors/circuits interface units which are responsible for digitizing the sensed data and also transforming the processed data into an analog signal. Since energy-efficiency is one of the most essential requirements of stand-alone, battery-operated IoT devices, the need for low-power data converters is increasing. Although data converters based on the existing complementary metal-oxide-semiconductor (CMOS) technology offer high sampling rates, they are energyinefficient due to the subthreshold leakage of CMOS transistors and scaling issues. Micro-electromechanical systems (MEMS) technology, in particular, provides an energy-efficient alternative to CMOS, while operating at low-to-medium speeds, required by IoT sensors and gadgets [1].

\section{MEMS-based Data Converters}

Multiple MEMS-based DACs have been illustrated using relays [2], resonators [3, 4], electrostatic actuators [5], and bent-beam electro-thermal actuators[6]. MEMS-based $\mathrm{ADCs}$, on the other hand, are generally more difficult to implement. In [7], a low-power MEMS ADC was illustrated using piezoelectric actuators with different lengths and normally-open contacts. The piezoelectric ADC produces a thermometer code at the output which requires additional circuits to obtain the final binary bits. A NEM relay-based ADC was presented in [2], in which a thermometer code is produced at the output using a bank of relays, designed to have different pull-in voltages. A relay- based decoder design was also proposed to obtain the final binary number. However, relays suffer from high contact resistance, wearing, and reliability issues [8]. The mechADC presented in [9] consists of a spring-guided slider with geometric coding of the output bits and flexible electrodes. Although the proposed design produces the output binary bits directly without the need for additional decoders, it suffers from contact and reliability issues due to the repeated sliding of the teeth electrodes against the output counter electrodes. In [10], a flash-style NEM resonator-based N-bit ADC was presented which required $2^{\mathrm{N}}-1$ resonators, operating at different drive frequencies, to generate a thermometer code at the output and an additional encoder is required to generate the final binary output bits. The non-contact-based operation of the resonators increases their reliability and durability compared to contact-based devices such as relays. In this work, the design proposed in [10] is modified to produce the output bits directly using only $\mathrm{N}$ resonators and multi-frequency excitation, thereby exponentially reducing the number of required devices. This modification leads to a huge reduction in circuit complexity and chip area. Furthermore, the proposed device is designed in a symmetric way to cancel the parasitic feedthrough signal due to the parasitic capacitance between the drive and sense electrodes and interconnects.

The paper is organized as follows: It starts by explaining the proposed device design and the feedthrough signal cancellation scheme, followed by illustrating the operation and the system architecture of the proposed resonator-based 3-bit ADC. Next, the experimental results are shown followed by a discussion about different aspects of the ADC such as sampling speed, and energy.

\section{DEVICE STRUCTURE AND OPERATION AND ADD SYSTEM ARCHITECTURE} Single Device Design

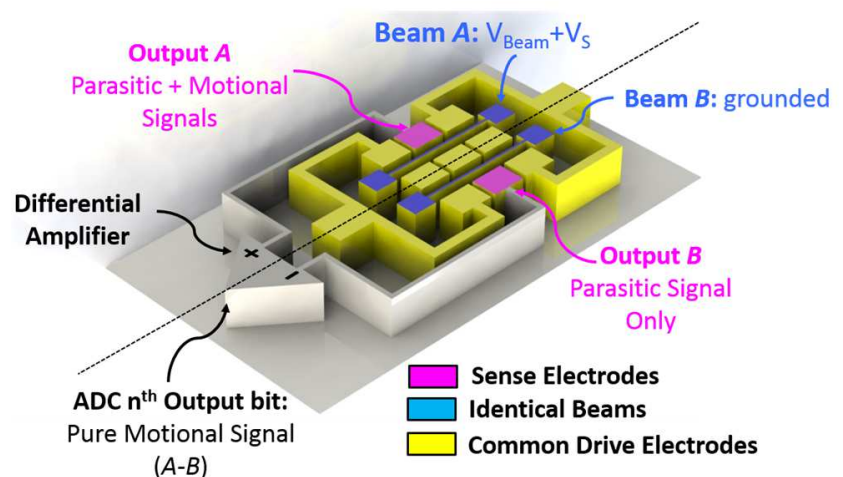

Figure 1: A 3D schematic of the device that generates one bit of the proposed ADC. The device is symmetric around the dashed line to ensure the proper cancellation of the parasitic signals coming from all shared drive electrodes. 
Figure 1 shows a 3D schematic of the device that generates one bit of the proposed ADC. Each device consists of two clamped-clamped micro-beams $A$ and $B$, highlighted in blue in Fig.1. Beam $A$ is biased with a DC voltage $V_{\text {Beam }}$ in addition to the sampled analog signal $V_{S}$. Beam $A$ is expected to resonate when actuated by a timevarying force with a frequency near its resonance frequency, while beam $B$ is grounded and remains unactuated. The two pink electrodes denoted as output $A$ and output $B$ in Fig.1, are used as sense electrodes. The five yellow electrodes, shared between the two beams as shown in Fig.1, can be used as drive electrodes, where multiple ac or dc signals can be applied. The feedthrough signal is the parasitic signal due to the capacitance between the drive and sense electrodes, and also due to the metal interconnects. By designing a fully-symmetric (mirrored) device as shown in Fig.1, it is expected that the parasitic capacitance between all shared drive electrodes and output $A$ is almost identical to that between the drive electrodes and output $B$. Hence, equal feedthrough signals are sensed at output $A$ and output $B$. In addition to the parasitic signal, output $A$ also contains another component, which is the motional signal resulting from the movement of beam $A$ near resonance. A differential amplifier is used to extract the motional signal by subtracting output $B$ from output $A$. The idea of using a symmetric device is similar to using an additional dummy resonator [11]. The main difference is that in the latter, a splitter per input is used to provide two input signals, equal in magnitude and $180^{\circ}$ out of phase, to the main and the dummy resonators, and their outputs can be combined directly to obtain the motional signal (the outputs can be collected from a single shared electrode). In our proposed design, the inputs, on the other hand, are shared between the resonators and the outputs are subtracted by a differential amplifier. The effectiveness of the proposed design becomes more pronounced for devices that need multiple drive electrodes such as logic gates, multi-frequency excitation-based devices, as a single differential amplifier is required to cancel the feedthrough signal, regardless of the number of inputs, while the design in [11] requires an additional splitter for each additional input. Furthermore, physically sharing drive electrodes guarantees, to a large extent, the full cancellation of the parasitic signal, saves the device area, and also makes the design more compact.

\section{ADC Concept}

As mentioned earlier, the concept of the proposed ADC is similar to the one presented in [10] in the way the sampled analog input $\left(V_{S}\right)$ is applied and how it changes the resonance frequency of the beam using the electrostatic softening effect. The difference is that instead of generating a thermometer code, the output bits are directly obtained using N-resonators only. This has been achieved by finding a relationship between the ADC input voltage and the final output bits values and using multi-frequency excitation. Figure 2 illustrates the relationship between the input voltage of a 3-bit ADC (top axis), the expected output bits values within each voltage range, and the resonance peaks at the edge of each voltage range. The input voltage range of the analog signal is from $0 \mathrm{~V}$ to $V_{D D}$. For a 3-bit ADC, the voltage range is divided into 8 equal segments, with a voltage step of $V_{D D} / 8$. Resonance peaks are obtained for the voltages that define the start and end of each voltage range. As the voltage increases, the peaks shift to the left due to the electrostatic softening effect. The intersection point of each two consecutive resonance peaks occurring at input voltages $V_{1}$ and $V_{2}$ defines the frequency of operation that covers that voltage range $\left(V_{2}-V_{l}\right)$. For example, in Fig.2, the intersection between the peak of $V_{D D} / 8$ and $V_{D D} / 4$ is denoted as $f_{1}$. By applying a drive signal with a frequency $f_{i}$ to the resonator, any input voltage that lies between $V_{D D} / 8$ and $V_{D D} / 4$ will generate a high output. By looking into Fig.2, we find out that each bit is ' 1 ' 'in 4 cases ( 4 voltage ranges). For example, $D_{0}$ is ' 1 ' if the input is within the following ranges: $V_{D D} / 8$ to $V_{D D} / 4,3 V_{D D} / 8$ to $V_{D D} / 2,5 V_{D D} / 8$ to $3 V_{D D} / 4$, and $7 V_{D D} / 8$ to $V_{D D}$. Therefore, by applying the following frequencies $f_{1}, f_{3}, f_{5}$, and $f_{7}$ to a single resonator, $D_{0}$ is generated, as shown in Fig.3. Similarly, $D_{l}$ is obtained by applying $f_{2}, f_{3}, f_{6}$, and $f_{7}$, while $D_{2}$ is obtained by applying $f_{4}, f_{5}, f_{6}$, and $f_{7}$.

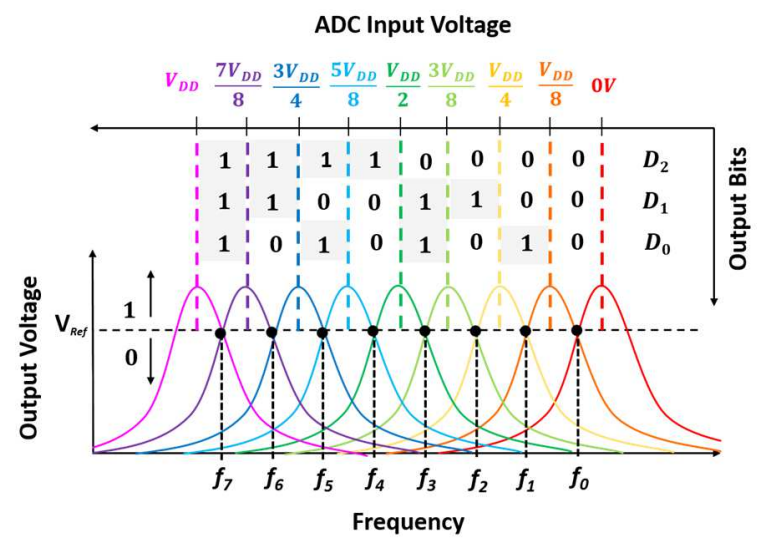

Figure 2: Operation of the proposed 3-bit ADC. The ADC input voltage $V_{S}$ is applied to the beam. As $V_{\text {S }}$ increases, the resonance peak shifts to the left. Each frequency of operation, located at the intersection point of two consecutive bits, covers a specific voltage range. From this figure, the frequencies of operations where a specific bit should be ' 1 ' are extracted and applied to a single resonator to generate that bit.

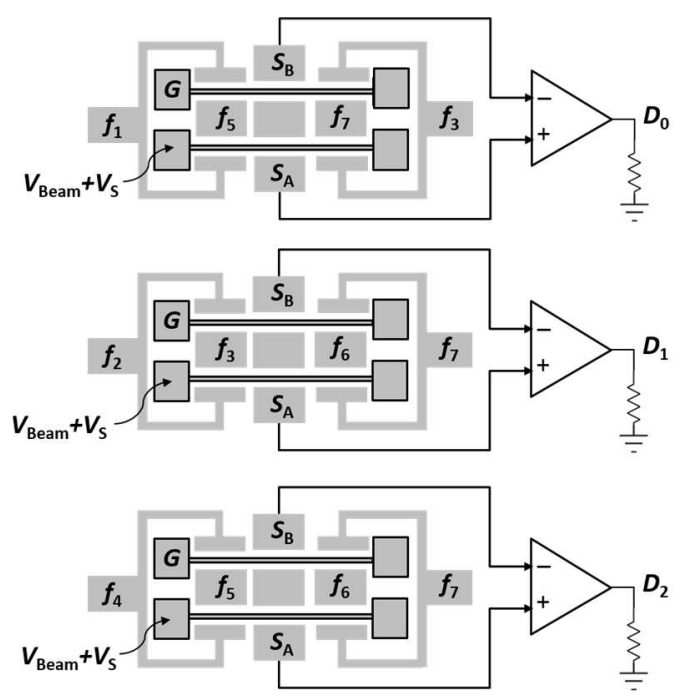

Figure 3: System Architecture of the proposed 3-bit resonator-based ADC. Each bit is generated by one resonator by applying all frequencies that lie within the voltage ranges where that bit is ' 1 '. 


\section{EXPERIMENTAL RESULTS}

Figure 4 shows a scanning electron microscopy (SEM) image of the tested device and the experimental setup. The tested silicon resonators were fabricated by MEMSCAP foundry using the SOIMUMPs process. The experiment was conducted in a vacuum chamber at room temperature.

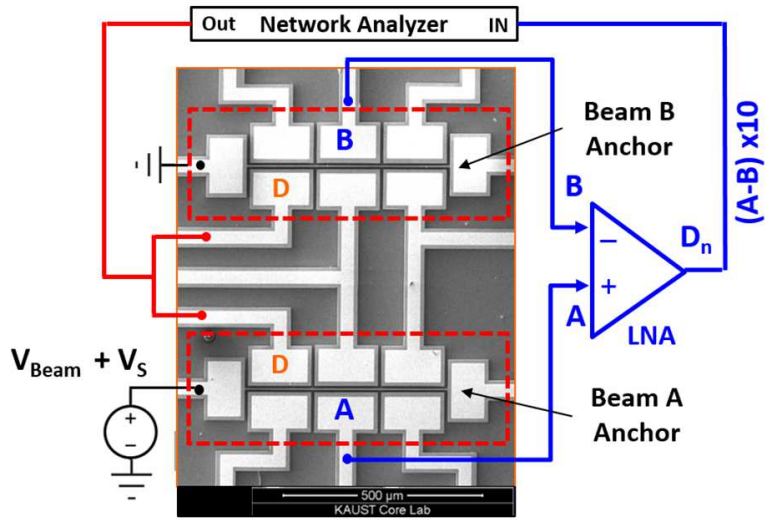

Figure 4: An SEM image of the tested device with the experimental setup. The beams are $500 \mu \mathrm{m}$ long, $3 \mu \mathrm{m}$ wide, $25 \mu \mathrm{m}$ thick, and separated from the side electrodes by $6 \mu \mathrm{m}$ airgaps. $V_{\text {Beam }}$ is $40 \mathrm{~V}$. A low noise amplifier (LNA) amplifies the motional signal $(A-B)$ by 10 times.

\section{Feedthrough Signal Cancellation}

The first step is to make sure that the feedthrough signal is properly canceled. As shown in Fig.4, beam $A$ is biased with a DC voltage, which is $40 \mathrm{~V}$ in the conducted experiment. As explained earlier, output $A$ contains the feedthrough signal and the motional signal while output $B$ contains the feedthrough signal only. A single electrode, denoted by $D$ in Fig.4, was used as the drive electrode as the network analyzer can provide a single output only. All other electrodes were grounded. Figure 5 shows the effect of canceling the feedthrough signal. The top part shows the output of resonator A (in red) without feedthrough signal cancellation which is indicated by having resonance and anti-resonance peaks [11]. The blue line shows the output $B$ signal which contains the feedthrough signal component only. The final output of the differential amplifier (A-B) is shown in green, indicating full cancellation of the feedthrough signal by getting rid of the anti-resonance peak and increasing the difference between the peak level and noise floor from $0.5 \mathrm{~dB}$ to $15 \mathrm{~dB}$.

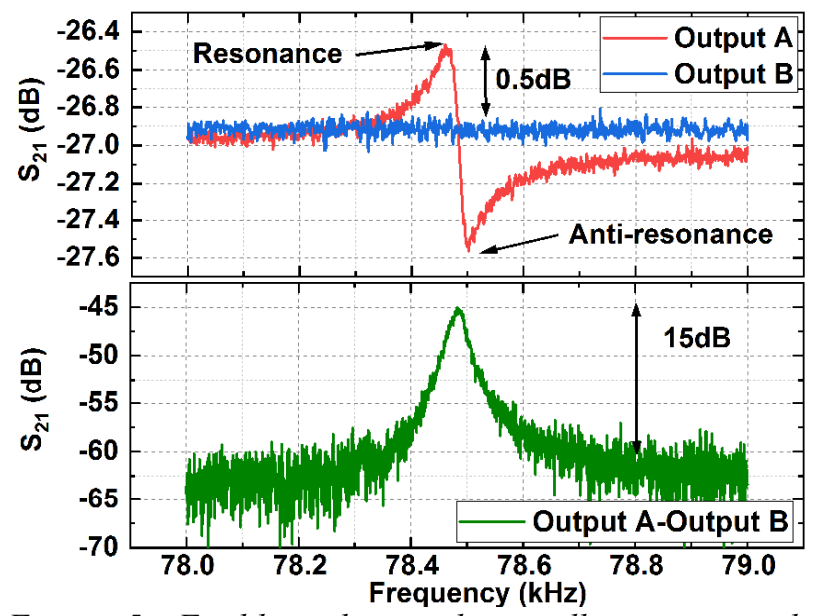

Figure 5: Feedthrough signal cancellation using the proposed device at 20 mTorr, using -34dBm input power.

\section{Frequency Response}

After canceling the feedthrough signal and obtaining the Lorentzian shape of the resonance peak, the peaks for the voltages at the start and end of each range are obtained. The analog signal can take any value between $0 \mathrm{~V}$ and $4 \mathrm{~V}$ $\left(V_{D D}\right)$. Figure 6 shows the frequency peaks for edge voltages and the frequencies of operations as well $\left(f_{l}\right.$ to $\left.f_{7}\right)$.

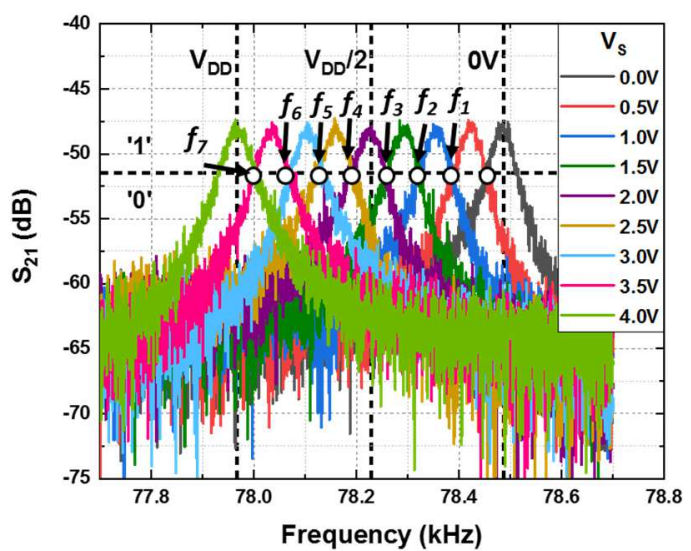

Figure 6: Frequency response of the resonator for different sampled input voltage values. The initial beam voltage is $40 \mathrm{~V}$ and $V_{S}$ is added to the beam voltage. The testing was done with -34dBm ac input power at $260 \mathrm{mT}$.

\section{Time Response}

After determining the frequencies of operation from Fig.5, time response is carried out to obtain the output bits. To obtain $D_{2}$, the frequencies $f_{4}, f_{5}, f_{6}$, and $f_{7}$ are applied sequentially to the drive electrode $D$, and the input voltage on the beam was swept from $0 \mathrm{~V}$ to $4 \mathrm{~V}$ using a voltage step $0.15 \mathrm{~V}$ and the resonator output was recorded. $D_{l}$ and $D_{0}$ were obtained similarly as shown in Fig.7.
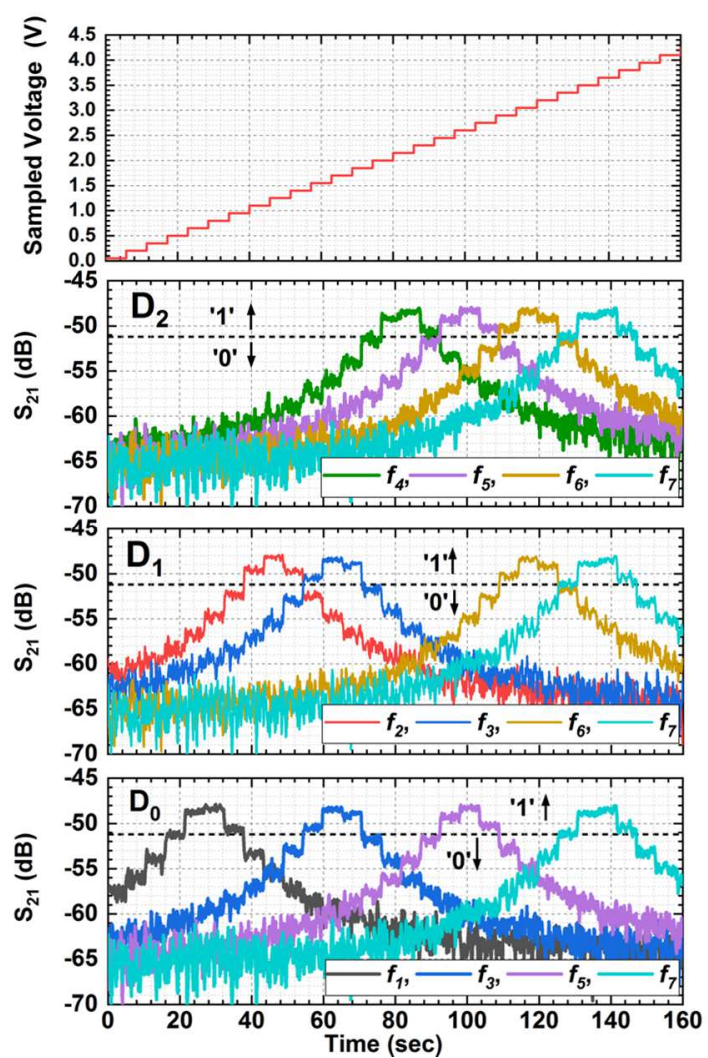

Figure 7: The top figure shows the ADC input voltage, followed by the output bits $D_{2}, D_{1}$, and $D_{0}$ in order. 


\section{DISCUSSION}

The MEM/NEM resonator technology provides interesting solutions for circuit designers and also has some limitations. In this part, we will discuss various aspects such as ADC sampling rate and energy consumption.

The sampling rate of the proposed resonator-based $\mathrm{ADC}$ is a function of the quality factor of the resonator $(Q)$ and its resonance frequency $f($ Speed $=f / Q)$. The measured $Q$ of the tested resonator is around 1348, the average resonance frequency is $78.2 \mathrm{kHz}$. This yields a sampling rate of $58 \mathrm{~S} / \mathrm{s}$. For a scaled resonator with the following dimensions (480 $\mathrm{nm}$ long, $40 \mathrm{~nm}$ wide, and $40 \mathrm{~nm}$ thick beam separated from side electrodes by $50 \mathrm{~nm}$ airgaps), the obtained resonance frequency is around $1.3 \mathrm{GHz}$. With an estimated maximum $Q$ of 1102 , a sampling rate of 1.19 $\mathrm{MS} / \mathrm{s}$ is attainable.

The energy consumption of the proposed 3-bit ADC is estimated to be $3.89 \mathrm{pJ}$ per conversion step. Table 1 compares different aspects of the tested, scaled resonatorbased $\mathrm{ADC}(3,4$, and 5 bits), the NEM resonator ADC presented in [10], and the CMOS ADC presented in [12]. Three orders of magnitude reduction in energy/conversion step and 6 orders of magnitude higher speed are achieved by scaling down the resonator dimensions. Furthermore, the scaled device is less complex and consumes less energy compared to the CMOS ADC presented in[12] and the NEMS resonator ADC presented in [10].

Table 1: Performance Comparison

\begin{tabular}{|c|c|c|c|c|}
\hline Device & $\begin{array}{l}\text { Resolution } \\
\text { (Bits) }\end{array}$ & $\begin{array}{c}\text { Device } \\
\text { Count }\end{array}$ & $\begin{array}{c}\text { Speed } \\
(\mathrm{S} / \mathrm{s})\end{array}$ & $\begin{array}{c}\text { FOM (fJ/ } \\
\text { Conv. } \\
\text { step) }\end{array}$ \\
\hline \multirow{3}{*}{$\begin{array}{c}\text { Tested } \\
\text { MEMS } \\
\text { ADC }\end{array}$} & 3 & 3 & \multirow{3}{*}{58} & 3897 \\
\hline & 4 & 4 & & 4567 \\
\hline & 5 & 5 & & 5335 \\
\hline \multirow{3}{*}{$\begin{array}{c}\text { Scaled } \\
\text { NEMS } \\
\text { ADC }\end{array}$} & 3 & 3 & \multirow{3}{*}{$\begin{array}{c}1.19 \\
\mathrm{M}\end{array}$} & 1.9 \\
\hline & 4 & 4 & & 2.96 \\
\hline & 5 & 5 & & 5.11 \\
\hline \multirow{2}{*}{$\begin{array}{c}\text { NEMS } \\
\text { ADC [10] }\end{array}$} & 3 & 7 & \multirow{2}{*}{$8 \mathrm{k}$} & 122 \\
\hline & 5 & 31 & & 44.46 \\
\hline $\begin{array}{c}\text { CMOS } \\
\mathrm{ADC}[12]\end{array}$ & 4 & 284 & $1.6 \mathrm{G}$ & 12.4 \\
\hline
\end{tabular}

\section{CONCLUSION}

In this paper, a compact MEM resonator-based ADC is presented. The proposed N-bit ADC requires $\mathrm{N}$ devices to generate the binary bits. The energy consumption of the scaled NEM resonator ADC is in femto joules per conversion step, operating at $1 \mathrm{MS} / \mathrm{s}$. We also verified the full cancelation of the feedthrough signal using the proposed design. Studying the effect of pressure and frequency variations on the ADC operation and linearity, and building a setup for multi-frequency excitation are currently under investigation.

\section{REFERENCES}

[1] D. Blaauw et al., "IoT design space challenges: Circuits and systems," in 2014 Symposium on VLSI Technology (VLSI-Technology): Digest of Technical Papers, 2014, pp. 1-2.

[2] R. Li and H. Fariborzi, "Ultra-Low Power Data
Converters with BEOL NEM Relays," in 2018 IEEE 61st International Midwest Symposium on Circuits and Systems (MWSCAS), 2018, pp. 627-630.

[3] S. Ahmed, X. Zou, and H. Fariborzi, "A MicroResonator Based Digital to Analog Converter for Ultralow Power Applications," in 2019 20th International Conference on Solid-State Sensors, Actuators and Microsystems \& Eurosensors XXXIII (TRANSDUCERS \& EUROSENSORS XXXIII), 2019, pp. 821-824: IEEE.

[4] S. Ahmed, X. Zou, N. Jaber, M. I. Younis, and H. Fariborzi, "A Low Power Micro-Electromechanical Resonator-Based Digital to Analog Converter," Journal of Microelectromechanical Systems, vol. 29, no. 3, pp. 320-328, 2020.

[5] H. Toshiyoshi et al., "Microelectromechanical digitalto-analog converters of displacement for step motion actuators," Journal of Microelectromechanical Systems, vol. 9, no. 2, pp. 218-225, 2000.

[6] P. Pandiyan, G. Uma, and M. Umapathy, "Design and Simulation of MEMS-Based Digital-to-Analog Converters for In-Plane Actuation," Arabian Journal for Science and Engineering, vol. 42, no. 12, pp. 49915001, 2017/12/01 2017.

[7] R. Proie, J. S. Pulskamp, R. G. Polcawich, T. Ivanov, and M. Zaghloul, "Low-power 3-bit piezoelectric MEMS analog to digital converter," in 2011 IEEE 24th International Conference on Micro Electro Mechanical Systems, 2011, pp. 1241-1244.

[8] A. C. David, D. N. Christopher, W. D. Christopher, A. P. Gary, M. K. Garth, and D. C. William, "Lifetime limitations of ohmic, contacting RF MEMS switches with $\mathrm{Au}, \mathrm{Pt}$ and $\mathrm{Ir}$ contact materials due to accumulation of 'friction polymer' on the contacts," Journal of Micromechanics and Microengineering, vol. 22, no. 10, p. 105005, 2012.

[9] P. Schmitt and M. Hoffmann, "How to Defeat Electric Noise in Measurement Acquisition Using a Micromechanical Analog-to-Digital Converter," in 2019 20th International Conference on Solid-State Sensors, Actuators and Microsystems \& Eurosensors XXXIII (TRANSDUCERS \& EUROSENSORS XXXIII), 2019, pp. 2001-2004.

[10]X. Zou, S. Ahmed, S. Kazmi, P. d. Costa, M. Younis, and H. Fariborzi, "A Nanoelectromechanical Resonator-Based Flash Style Analog to Digital Converter," in 2020 IEEE 33rd International Conference on Micro Electro Mechanical Systems (MEMS), 2020, pp. 822-825.

[11] J.-Y. Lee and A. Seshia, "Parasitic feedthrough cancellation techniques for enhanced electrical characterization of electrostatic microresonators," Sensors and Actuators A: Physical, vol. 156, no. 1, pp. 36-42, 2009.

[12]A. S. Almansouri, A. Alturki, H. Fariborzi, K. N. Salama, and T. Al-Attar, "A 12.4 fJ-FoM 4-Bit Flash ADC Based on the StrongARM Architecture," in 2018 14th Conference on Ph. D. Research in Microelectronics and Electronics (PRIME), 2018, pp. 37-40: IEEE.

\section{CONTACT}

*Sally Ahmed, sally.ahmed@kaust.edu.sa 